7. Healy B (1992) Women in science: From panes to ceilings. Science $255: 1333$

8. Homans H (1987) Man-made myths: The reality of being a woman scientist in the NHS. In: Spencer A, Padmore D (eds). In a man's world. Tavistock Publications, London, p 87112

9. Landeshauptstadt München, Personal- und Organisationsreferat (Hrsg) Frauenberichte 1992

10. Mason J (1991) The invisible-obstacle race. Nature 353:205-206

11. Onnen-Isemann C, OBwald U (1991) Aufstiegsbarrieren für Frauen im Universitätsbereich. Schriftenreihe Studien zu Bildung und Wissenschaft, Band 99. Hrsg vom Bundesminister für Bildung und Wissenschaft. Bock, Bad Honnef

12. Osborn M (1992) Prospects for women in science. Nature $360: 101$

13. Osborn M (1994) Status and prospects of women in science in Europe. Science $263: 1389-1391$

14. Sieverding $M$ (1990) Psychologische Barrieren in der beruflichen Entwicklung von Frauen. Das Beispiel der Medizinerinnen. Enke, Stuttgart

15. Symonds A (1983) Emotional conflicts of the career woman: Women in medicine. Amer J Psychoanalysis $43: 21-37$

16. Wermuth N (1992) Frauen an Hochschulen. Statistische Daten zu den Karrierechancen. Schriftenreihe Studien zu Bildung und Wissenschaft, Band 105. Hrsg vom Bundesminister für Bildung und Wissenschaft (Mitautoren Hardt J, Prawitz I, Streit R). Bock, Bad Honnef

17. Wetterer A (Hrsg) (1992) Profession und Geschlecht. Über die Marginalität von Frauen in hochqualifizierten Berufen. Campus, Frankfurt New York

18. Wobbe Th (1992) Wer hat Angst vor Frauenquoten? Anmerkungen zur Marginalisierung von Wissenschaftlerinnen. Sozialwissenschaften und Berufspraxis (SUB) $15: 359-375$

\title{
Psychologische und soziale Karrierehemmnisse im Berufsweg der Ärztin
}

\section{Sieverding}

Für die bereits vielfach dokumentierte unbefriedigende berufliche Situation von Medizinerinnen (Kaul-Hecker \& Meyer, 1991; Mesletzky, 1994; Sieverding, 1990) sind eine Reihe von sozialen und psychologischen Faktoren verantwortlich, wobei diese nicht unabhängig voneinander zu sehen sind. Als Beispiele für soziale Karrierehemmnisse seien genannt:

Das Fehlen von adäquaten Kinderbetreuungseinrichtungen bei gleichzeitiger Delegation der Sorge fü die Kinder an die Frauen: Der ärztliche Beruf ist besonders schwer mit einer Familie vereinbar. Während die meisten Ärzte zu Hause cine Partner haben, die Haushalt und Kindererziehung übernimmt und die eigenen beruflichen Ambitionen ganz aufgibt oder zugunsten der Familie zurücksteckt, verfügen nur wenige Ärztinnen über einen solchen Partner. Wie eine Befragung an der Medizinischen Hochschule Hannover gezeigt hat, waren praktisch alle Partner der befragten Ärztinnen (92\%) ebenfalls voll berufstätig und überließen ihnen zudem den Hauptanteil an Haushalt und Kindererziehung (Kaul-Hecker \& Meyer, 1991).

Benachteiligungen von Medizinerinnen bei Einstellungen und Beförderungen: Bei Stellenbesetzungen werden häufig männliche Bewerber bevorzugt, da bei ihnen nicht mit einem Ausfall wegen Schwangerschaft und Erziehungsurlaub zu rechnen ist (s.z.B. Färber \& Jenschke, 1993). 
Fehlende Förderung der wissenschaftlichen Qualifikation und des beruflichen Aufstiegs von Medizinerinnen: Die medizinische Scientific Community ist von Männern dominiert; Medizinerinnen werden darin zu wenig gefördert; ein Großteil ihrer Arbeit besteht aus Routinearbeiten und Patientenversorgung (KaulHecker \& Meyer, 1991); Ärztinnen fehlen weibliche Rollenmodelle und Mentorinnen.

Einhergehend mit sozialen sind eine Reihe von psychologischen Karrierehemmnissen wirksam. Im folgenden möchte ich einige Ergebnisse dazu aus verschiedenen Untersuchungen mit männlichen und weiblichen Medizinstudierenden vorstellen (Sieverding, 1990, 1992, Sieverding \& Rauchfuß, 1993).

Es hat sich gezeigt, daß zu idealistische Erwartungen an den ärztlichen Beruf keine günstige psychologische Ausgangsbasis sind. Bei der Wahl des Medizinstudiums stehen idealistische und humanitäre Ziele - z.B. der Wunsch, Menschen zu helfen oder die Arzt-Patienten-Beziehung zu verbessern - eindeutig im Vordergrund. Bei der Konfrontation mit der Realität des Klinikalltages zeigt sich spätestens im Praktischen Jahr, daß die bürokratischen und hierarchischen Strukturen nur wenig Spielraum für die Umsetzung solcher Ziele bieten. Die im Vergleich zu ihren männlichen Kommilitonen noch höher motivierten und ehrgeizigen Studentinnen werden dementsprechend stärker von einer tiefgreifenden Enttäuschung erfaßt, ein Phänomen, das in der Literatur auch für andere Berufe als Praxisschock oder Berufseintritts-Schock beschrieben wurde. Fehlende Strategien im Umgang mit dem Berufseintritts-Schock verstärken das Problem. Während es Männern leichter zu fallen scheint, ihre ursprünglichen Ansprüche auf ein realistisches $\mathrm{Maß}$ herunterzuschrauben, reagieren nicht wenige Medizinerinnen entsprechend einem Alles-oder-Nichts-Prinzip: „Hier ist alles so schrecklich, damit will ich nichts zu tun haben." Es ist allerdings zu bedenken, daß ihren männlichen Kollegen keine Alternative zur Anpassung bleibt. Während Frauen nach wie vor zur Berufsrolle die Alternative der Hausfrauen- und Mutterrolle angeboten wird, und zwar verstärkt in Zeiten angespannter Arbeitsmarktstrukturen, können Männer (noch) nicht von dem Modell einer lebenslangen Vollzeiterwerbstätigkeit abweichen, ohne gesellschaftlich mißachtet zu werden. Dies wurde beispielsweise in der „Hausmänner-Studie" von Strümpel und Kollegen eindrucksvoll belegt (Strümpel et al., 1988). Darin wird unter anderem ein Chefarzt zitiert, der auf den Wunsch eines ihm unterstellten Arztes nach Teilzeitarbeit antwortet: „Ich häng mich auf, wenn solche Sitten eingeführt werden" (ebd., S. 89).

Der Konflikt zwischen Kind und Beruf ist für Medizinerinnen besonders schwierig zu lösen, da sie kaum aufKompromißlösungen, wie in vielen typischen Frauenberufen möglich, zurückgreifen können. Nur selten ist eine Reduzierung der Arbeitszeit möglich, und wenn, dann oft nur unter Verzicht auf eine weitere berufliche Qualifizierung. Problematisch ist auch die Unterbrechung der Berufstätigkeit insbesondere, wenn diese gleich nach $\mathrm{Abschluß} \mathrm{des} \mathrm{Studiums} \mathrm{oder} \mathrm{nach} \mathrm{nur} \mathrm{kurzer}$ Berufstätigkeit erfolgt Je länger der Ausstieg dauert, desto geringer werden die Chancen, je wieder in den ärztlichen Beruf, insbesondere aber in die Klinik hineinzukommen. Verschärft wird dieser Konflikt durch die gerade in Westdeutschland besonders ausgeprägte Mutter-Kind-Ideologie, die die Berufstätigkeit von Müttern kleiner Kinder verteufelt und so beruflich engagierte Frauen mit Kindern in Gewissenskonflikte stürzt. Obwohl inzwischen in vielen wissenschaftichen 
Studien eindeutig belegt werden konnte, daß die Berufstätigkeit von Müttern kleinen Kindern nicht schadet, sondern im Gegenteil häufig sehr positive Auswirkungen auf das Wohlergehen von Müttern und Kindern hat (einen Überblick gibt Fthenakis, 1989), hält sich dieses Vorurteil hartnäckig: leider nicht nur in der Boulevardpresse, sondern zum Teil auch in ärztlichen Fachzeitschriften. Und so empfiehlt Fthenakis, „,anstatt weiter danach zu fragen, ob sich die mütterliche Berufstätigkeit auf die kindliche Entwicklung beeinträchtigend auswirkt, eher nach den Bedingungen zu fragen, die es Frauen in unserem Lande so schwer machen, Berufs- und Familientätigkeit miteinander zu vereinbaren. In dieser Hinsicht ist Deutschland im Vergleich zu anderen Ländern mit hoher Muttererwerbstätigkeit noch ein Entwicklungsland" (Fthenakis, 1989, S. 20).

Die Identifikation mit traditionellen Geschlechtsrollenerwartungen erweist sich als eine besonders gravierende psychologische Barriere für eine berufliche Karriere im Krankenhaus. Je mehr eine Frau sich mit der traditionellen farniliären Aufgabenteilung, wonach eine Frau primär für Kindererziehung und Haushalt zuständig ist, identifiziert, um so schwerer wird es ihr fallen, von ihrem Partner eine stärkere Beteiligung an diesen Aufgaben einzufordern bzw. eine außerfamiliale Betreuung zu organisieren. Erschwert wird der unausweichliche Konflikt zwischen den Partnern durch die Unantastbarkeit der männlichen Geschlechtsrolle. Die beiden Geschlechtsrollen sind eng aufeinander bezogen, und eine tatsächliche Veränderbarkeit der weiblichen Rolle ist nur dann gegeben, wenn sich die männliche Rolle mitändert. Das heißt, eine wirkliche Beteiligung am Berufsleben ist der Frau nur möglich, wenn sich der Mann nicht nur in Lippenbekenntnissen, sondern tatsächlich an den Familienaufgaben beteiligt. Dies würde jedoch erfordern, daß er seinerseits sein berufliches Engagement reduziert. Bisher stellen jedoch die wenigsten Männer ihre Berufsrolle in Frage, und kaum einer verspürt Lust, den häuslichen Bereich zu ,erobern“".

Abschließend möchte ich noch auf eine Variable eingehen, die ich in meinen Untersuchungen schwerpunktmäßig analysiert habe, nämlich die Bedeutung von subjektiven Berufskonzepten für den ärztlichen Beruf. Nach der Selbstkonzepttheorie der beruflichen Entwicklung von Donald Super (Super et al., 1963) suchen Menschen sich einen solchen Beruf aus, von dem sie glauben, daß sie das geeignete Persönlichkeitsprofil mitbringen. Verschiedene Untersuchungen haben gezeigt, daß Personen um so erfolgreicher und zufriedener in einem Beruf sind, je besser ihr subjektives Berufskonzept und ihr Selbstkonzept übereinstimmen. Welche Persönlichkeitseigenschaften sind notwendig für den ärztlichen Beruf? Zur Erfassung der subjektiven ärztlichen Berufskonzepte verwandte ich eine Eigenschaftsliste, die zwei Skalen enthält, die als typisch für das weibliche bzw. männliche Geschlecht gelten, sowie zwei Skalen zum Leistungsstreben und zur Selbstbehauptung. Die Maskulinitäts- bzw. Instrumentalitätsskala des Personal Attributes Questionnaire von Spence und Helmreich (1978) enthält aufgabenbezogene Eigenschaften wie selbstsicher, durchsetzungsfähig, aktiv, leicht Entscheidungen fällend. Die Feminitäts- oder Expressivitätsskala enthält Eigenschaften, die der sozialemotionalen Unterstïtzung anderer dienen, wie hilfsbereit, einfühlsam, freundlich. In der aufgrund von Vorversuchen konstruierten Selbstbehauptungsskala sind ebenfalls instrumentelle Eigenschaften enthalten, die einen eher negativen Beigeschmack haben, wie offensiv, cool oder egoistisch. Es zeigt sich nun, 
daß es für den ärztlichen Beruf (mindestens) zwei subjektive Berufskonzepte gibt. Fragt man Medizinstudierende, welche Eigenschaften der ideale Arzt/die ideale Ärztin im Umgang mit Patienten haben sollte, stehen feminine/expressive Eigenschaften an erster Stelle. Geht es jedoch darum, welche Eigenschaften förderlich sind, um im Krankenhaus eine Stelle zu bekommen und aufzusteigen, d.h., welche Eigenschaften der Karriere im Krankenhaus förderlich sind, wird eine ganz andere Rangreihenfolge aufgestellt. Dann steht Leistungsorientierung an erster Stelle, gefolgt von instrumentellen/maskulinen Eigenschaften und Eigenschaften der Selbstbehauptung; expressive/feministische Eigenschaften werden als am wenigsten förderlich eingeschätzt, und zwar von männlichen wie weiblichen Medizinstudierenden. Wie unterschiedlich die beiden subjektiven Berufskonzepte des ärztlichen Berufs sind, ist in Abb. 1 dargestellt.

Bei der Entscheidung für das Medizinstudium orientieren sich junge Männer und Frauen fast ausschließlich an dem Idealkonzept ärztlicher Tätigkeit, welches auch ihrem Selbstkonzept sehr viel ähnlicher ist als das Karrierekonzept. Vergleicht man das Selbstkonzept und das Karrierekonzept von Studienanfängern und Studierenden im Praktischen Jahr, zeigt sich ein auffallender Geschlechtsunterschied: am Ende des Studiums weisen die PJlerinnen eine signifikant größere Diskrepanz zwischen ihrem Selbstkonzept und dem Karrierekonzept auf, und zwar sowohl im Vergleich zu ihren männlichen Kommilitonen als auch im Vergleich zu Medizinstudentinnen am Anfang des Studiums (s. Abb. 2).

\section{Karrierekonzept und Idealkonzept des ärztlichen Berufs im Vergleich}

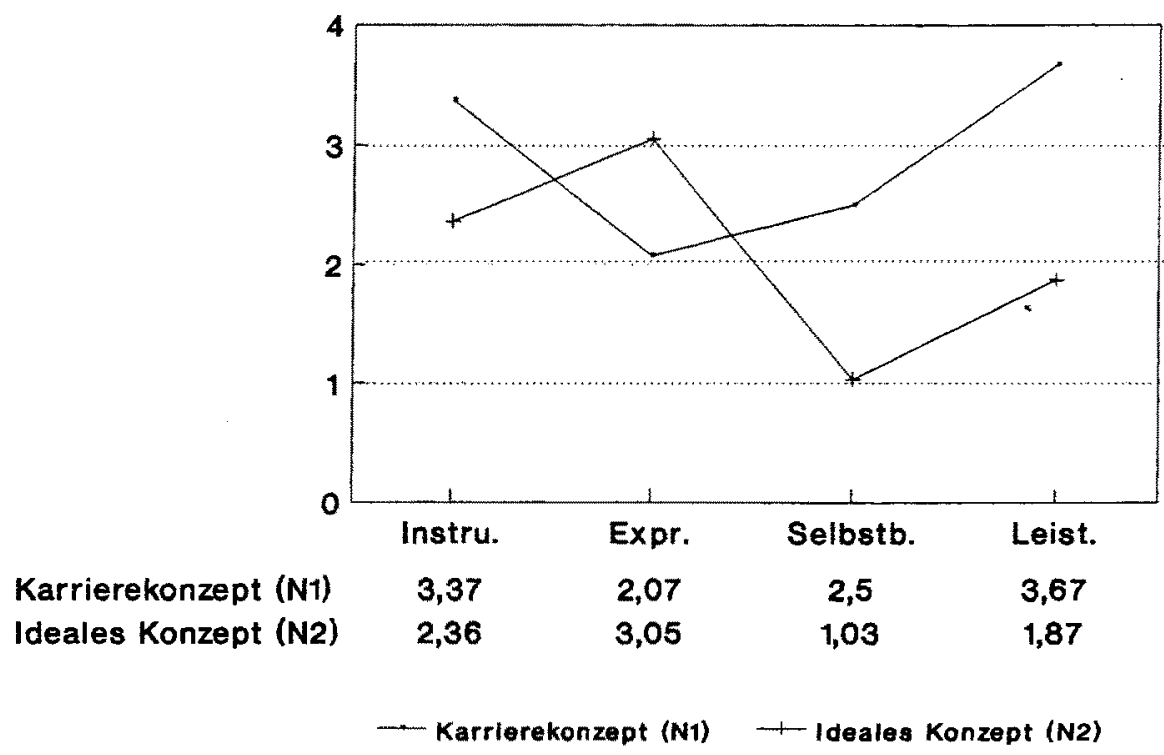

Abb. 1. Karrierekonzept und Idealkonzept bei Studienanfängern ( $11=221, \mathrm{~N} 2=90)$, Skalenmittelwerte $(4=$,sehr förderlich“ $)$ 


\section{Gesamtdiskrepanz zwischen Karrierekonzept und Selbstkonzept}

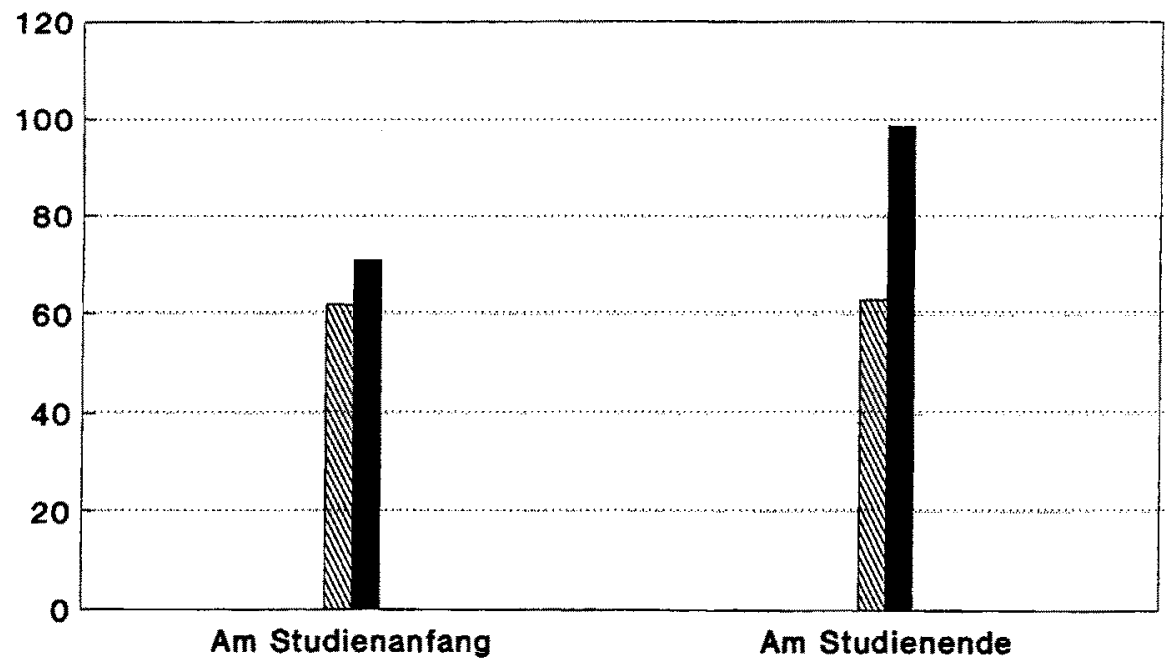

\section{Männer Frauen}

Abb. 2. Gesamtdiskrepanz zwischen Karrierekonzept und Selbstkonzept, bei Männern und Frauen am Anfang und am Ende des Medizinstudiums $(n=450)$, arithmetische Mittelwerte

Diese durchschnittlich deutlich größere Diskrepanz entsteht durch zwei Phänomene: einerseits haben die PJlerinnen ein besonders maskulines Karrierekonzept, d.h. sie haben den Eindruck, daß feminine Eigenschaften überhaupt nicht zählen. So beschreiben einige ihre Erfahrungen in der Klinik, daß sie als ,Schwester" angesprochen werden, wenn sie zu mitfühlend sind. Andererseits wiesen sie ein recht feminines Selbstkonzept auf. Je femininer sich die Medizinerinnen beschreiben, desto geringer sind ihr berufliches Selbstvertrauen sowie ihre Hoffnungen, daß sie ihre Karrierepläne verwirklichen können. Dagegen bringen solche Medizinerinnen, die sich selbst als hochinstrumentell und leistungsorientiert beschreiben, genauso gute psychologische Voraussetzungen für eine Karriere am Krankenhaus mit wie ihre männlichen Kommilitonen. Die Identifikation mit typisch femininen Eigenschaften im Selbstkonzept erweist sich somit als eine weitere psychologische Barriere für ein berufliches Fortkommen an der Klinik für Ärztinnen. Interessanterweise reagieren nicht wenige Medizinerinnen auf die maskulin dominierten und geprägten Strukturen in der Klinik nicht mit einer Anpassung, sondern sogar eher in Richtung einer besonderen Betonung ihrer femininen Werte. Dieser Prozeß geschieht sicher nur zum Teil bewußt und unter dem Druck einer Unwelt, die Frauen immer wieder auf ,ihre" feminine Rolle mit den dazu assoziierten Eigenschaften festzulegen versucht. 


\section{Literatur}

Färber C \& Jenschke ML (Hrsg) (1993) Gleichstellungspolitik an Universitätsklinika. Dokumentation der 2. Jahrestagung der Kommission „Klinika“. Freie Universität Berlin

Fthenakis WE (1989) Mütterliche Berufstätigkeit, außerfamiliale Betreuung und Entwicklung des (Klein-)Kindes aus kinderpsychologischer Sicht. Zeitschrift für Familienforschung, $1: 5-27$

Kaul-Hecker U \& Mayer B (1991) Umfrage zur Situation wissenschaftlicher Mitarbeiterinnen und Mitarbeiter an der Medizinischen Hochschule Hannover (MHH) im Sommer 1991. Medizinische Hochschule Hannover

Mesletzky J (1994) Gleiche Rechte, gleiche Chancen? Berliner Ärzte, 11-14

Sieverding M (1990) Psychologische Barrieren in der beruflichen Entwicklung von Frauen. Das Beispiel der Medizinerinnen. Enke, Stuttgart

Sieverding M (1992) Berufskonzepte von Medizinstudierenden: Kongruenzen und Diskrepanzen zwischen Selbstkonzept, beruflichem Idealkonzept und Karrierekonzept. Zeitschrift für Arbeits- und Organistaionspsychologie, 157-166

Sieverding M \& Rauchfüß M (1993) Ärztliches Karrierekonzept und Selbstkonzept bei Medizinstudierenden in Ost- und West-Berlin. Zeitschrift für Medizinische Psychologie $2: 82-90$

Spence JT \& Helmreich RL (1978) Masculinity \& femininity. Their psychological dimensions, correlates \& antecedents. Austin: University of Texas Press

Strümpel B, Prenzel W, Scholz J \& Hoff A (1988) Teilzeitarbeitende Männer und Hausmänner. Motive und Konsequenzen einer eingeschränkten Erwerbstätigkeit von Männern. Edition Sigma, Berlin

Super DE, Starishevsky R, Matlin N \& Jordaan JP (Eds) (1963) Career development: Selfconcept theory. New York: College Entrance Examination Board

\section{Frauenärztin und akademische Karriere}

C. Нӧß

Seit Zulassung zur Habilitation in Deutschland im Jahre 1918 hat sich die Situation für Frauen nur unwesentlich geändert, nach wie vor finden sich wenige in den „obersten Etagen“ des von Männern dominierten Hochschulbetriebes [2, 12].

Trotz steigender Zahl der Medizinstudentinnen (Tabelle 1) gibt es noch keine entsprechende Zunahme des Frauenanteils in den höheren Positionen.

Noch immer klafft zwischen den 30\% - in der Gynäkologie sogar 60\% - weiblicher Assistenten und der mageren Zahl von 4-6\% bei Habilitation und Professur eine große Lücke. Trotz staatlich verordneter Frauenförderung finden sich lediglich 1,9\% C4-Professorinnen (Tabelle 2).

Vergleiche mit den USA bestätigen diese Situation [1], somit kann es kein Problem ausschließlich der Deutschen Geschichte sein.

Nach neueren amerikanischen Studien [3] erfüllen Ärztinnen und Wissenschaftlerinnen an Universitätskliniken die gleichen Aufgaben wie ihre männlichen Kollegen, sind ähnlich produktiv und veröffentlichen genauso viele Beiträge in Fachzeitschriften. Allerdings bekommen Frauen immer noch wenig soziale Anerkennung, werden langsamer befördert und beziehen in Relation zu gleichgestellten männlichen Kollegen ein geringeres Gehalt.

Offensichtlich bestehen Mechanismen, die den Anteil der Frauen an Spitzenpositionen niedrig halten, und Quotenregelungen sind anscheinend weder ausrei- 